\title{
Bekenstein bound from the Pauli principle
}

\author{
G. Acquaviva $\odot,{ }^{*}$ A. Iorio $\odot,{ }^{\dagger}$ and L. Smaldone ${ }^{*}$ \\ Faculty of Mathematics and Physics, Charles University, \\ V Holešovičkách 2, 18000 Praha 8, Czech Republic
}

(Received 8 June 2020; accepted 1 October 2020; published 2 November 2020)

\begin{abstract}
Assuming that the degrees of freedom of a black hole are finite in number and of fermionic nature, we naturally obtain, within a second-quantized toy model of the evaporation, that the Bekenstein bound is a consequence of the Pauli exclusion principle for these fundamental degrees of freedom. We show that entanglement, Bekenstein, and thermodynamic entropies of the black hole all stem from the same approach, based on the entropy operator whose structure is the one typical of Takahashi and Umezawa's thermofield dynamics. We then evaluate the von Neumann black hole-environment entropy and noticeably obtain a Page-like evolution. We finally show that this is a consequence of a duality between our model and a quantum dissipativelike fermionic system.
\end{abstract}

DOI: 10.1103/PhysRevD.102.106002

\section{INTRODUCTION}

This paper moves from the results of previous research [1] but reversing the point of view. There, Bekenstein's argument that a black hole $(\mathrm{BH})$ reaches the maximal entropy at disposal of a physical system (i.e., that it saturates the Bekenstein bound [2]), leads to two main proposals: i) the degrees of freedom (d.o.f) responsible for the BH entropy have to take into account both matter and spacetime and hence must be of a new, more fundamental nature than the d.o.f we know (with Feynman [3], here we call such d.o.f "Xons", see also [4] and [5]); ii) the Hilbert space $\mathcal{H}$ of the $X$ ons of a given $\mathrm{BH}$ is necessarily finite dimensional

$$
\operatorname{dim} \mathcal{H}=e^{\mathcal{S}_{\mathrm{BH}}},
$$

with $\mathcal{S}_{\mathrm{BH}}$ the Bekenstein entropy. With these, in [1] it was shown that the (average) loss of information is an unavoidable consequence of the nonvanishing relic entanglement between the evaporated matter and the spacetime.

In search of a unifying view of the various types of entropies involved in the $\mathrm{BH}$ evaporation (i.e., Bekenstein, thermodynamical, and entanglement entropies, see, e.g., [6]), here we reverse that logic. Namely, we start off by

\footnotetext{
"gioacqua@utf.troja.mff.cuni.cz

iorio@ipnp.troja.mff.cuni.cz

*smaldone@ipnp.mff.cuni.cz
}

Published by the American Physical Society under the terms of the Creative Commons Attribution 4.0 International license. Further distribution of this work must maintain attribution to the author(s) and the published article's title, journal citation, and DOI. Funded by SCOAP ${ }^{3}$. supposing that in a $\mathrm{BH}$ only free $X$ ons exist (hence, there can only be one kind of entropy at that level), and we suppose that they are finite in number and fermionic in nature. This amounts to having a finite dimensional $\mathcal{H}$. With these assumptions, here we show that the evaporation is a dynamical mechanism producing a maximal entanglement entropy, equal to the initial entropy of the $\mathrm{BH}$.

This is an instance of the Bekenstein bound, obtained here with arguments that do not assume preexisting geometrical (spatiotemporal) concepts. In fact, for a full identification with the standard formulae (see [7], where the bound is rigorously defined in quantum field theory (QFT) and, e.g., the review [8]) one needs to associate geometrical concepts to the Xons. For instance, one could make each d.o.f correspond to one elementary Planck cell. Nonetheless, in our picture we do not need the exact expression of the bound. What is crucial is that the Xons are taken to be finite in number and fermionic, otherwise the entanglement entropy would just indefinitely grow without reaching a maximal value. It is suggestive, though, that taking on board the geometric picture of $X$ ons as quanta of area (Planck cells), the horizon of the $\mathrm{BH}$ is of nonzero size as an effect of a Pauli exclusion principle. Before entering the details of what we just discussed, let us now briefly put our work into the context of current literature.

Bekenstein entropy $[9,10]$ is traditionally regarded as a measure of our ignorance about the d.o.f, which formed the $\mathrm{BH}$ [10-13], and as a consequence of the no-hair theorem [14]. However, other interpretations have been proposed in literature, as in Loop Quantum Gravity (LQG), where BH entropy is a counting of microstates corresponding to a given macroscopic horizon area $\mathcal{A}[15,16]$. Along these lines, Bekenstein proposed a universal upper bound for the entropy of any physical system contained in a finite 
region [2], which is saturated by BHs. This implies [17] that the entropy of every system in a finite region is bounded from above by the Bekenstein entropy of a $\mathrm{BH}$, whose horizon coincides with the area of the boundary of that region (see [7] and also [8]).

Using the approach of QFT in curved spacetimes, Hawking discovered the black body spectrum of $\mathrm{BH}$ radiation [18]. In the meantime, Umezawa and Takahashi developed their thermofield dynamics (TFD) [19] (see also Ref. [20]), that immediately appeared to be a fruitful tool to describe $\mathrm{BH}$ evaporation [21]. In [22], with the help of an entropy operator, whose structure is natural in TFD, the $\mathrm{BH}$-radiation entropy is viewed as an entanglement entropy of radiation modes with their "TFD-double" (the modes beyond the horizon).

Although the relation between QFT in curved spacetime and TFD was studied already in Refs. $[23,24]$, the renewed interest comes in connection with the AdS/CFT correspondence [25], where in a two-sided anti-de Sitter BH, the specular asymptotic region is mapped into two copies of a conformal field theory. The thermal nature of the $\mathrm{BH}$ is then naturally seen through TFD. Extensions to incorporate dissipative effects are in the recent [26,27].

Since a BH, initially described as a pure state, could end up in a mixed state (this is actually the view of [1]), questions arise on the unitary evolution, as first noticed by Hawking [28] and then extensively discussed, from different points of view, see e.g., Refs. [29-39]. In particular, in Refs. [31,40] Page studied the bipartite system BHradiation, in a random (Haar distributed) pure state, computing the radiation entanglement entropy as function of the associated thermodynamical entropy. He found a symmetric curve (Page curve) which goes back to zero when the BH is completely evaporated. In Ref. [36] he postulated that entanglement entropy, as a function of time, follows the minimum between Bekenstein and radiation thermodynamic entropy (Conjectured Anorexic Triangular Hypothesis). Recently [41], a Page curve was also derived from holographic computations [42].

As said, in this paper we reverse the line of reasoning of Ref. [1] and present a simple, purely quantum toy model of the dynamics of $\mathrm{BH}$ evaporation, focusing on the fundamental d.o.f. In Sec. II the basic assumptions are the finiteness of slots (quantum levels) available for the system and the fermionic nature of such d.o.f. The finiteness of the Hilbert space of states follows from the Pauli exclusion principle. In Sec. III we compute the von Neumann entropy of the subsystems during their evolution. This is remarkably given by the expectation value of the TFD entropy operator [22], and it has the same qualitative behavior of a Page curve: it starts from zero and ends in zero while its maximum is reached at half of the evaporation process. That maximum is identified here with the Bekenstein entropy of the $\mathrm{BH}$ at the beginning of the evaporation. We can, therefore, argue that Bekenstein bound itself descends from the Pauli principle. In Sec. IV we explain the relation with TFD by mapping our model to an equivalent description as a dissipativelike system. The last section is left to our conclusions, while in the Appendix we show the connection between TFD and von Neumann entropies in the present context.

Throughout the paper we adopt units in which $c=\hbar=1$.

\section{BASIC ASSUMPTIONS AND MODEL OF BH EVAPORATION}

We assume that the fundamental d.o.f are fermionic $(\mathrm{BH}$ models based on fermions are available in literature, see, e.g., the Sachdev-Ye-Kitaev model $[43,44])$. As a consequence, each quantum level can be filled by no more than one fermion. This assures that the Hilbert space $\mathcal{H}$ of physical states with a finite number of levels is finite dimensional. In fact, if the fundamental modes were bosons, then the requirement that the number of slots available were finite would not have been sufficient to guarantee the finiteness of $\operatorname{dim} \mathcal{H}$. Let us recall now that, in the picture of [1], it is only at energy scales below those of quantum gravity (e.g., at the energy scales of ordinary matter) that the field modes are distinguishable from those "making" the spacetime, hence we can write

$$
\mathcal{H}_{F} \otimes \mathcal{H}_{G} \subseteq \mathcal{H}
$$

Here $F$ and $G$ stand for "fields" and "geometry", respectively. In other words, at low energy, the $F$-modes will form quantum fields excitations, that is, the quasiparticles (from the $X$ ons point of view) immersed into the spacetime formed by the $G$-modes.

Now, say $N$ is the total number of quantum levels (slots) available to the $\mathrm{BH}$. The evaporation consists of the following steady process: $N \rightarrow(N-1) \rightarrow(N-2) \rightarrow \cdots$. That is, the number of free $X$ ons steadily decreases, in favor of the Xons that, having evaporated, are arranged into quasiparticles and the spacetime they live in. One might think of a counter that only sees free Xons and hence keeps clicking in one direction as the $\mathrm{BH}$ evaporates till its complete stop.

In this picture: i) there is no preexisting time because the natural evolution parameter is the average number of free $X$ ons; and ii) there is no preexisting space to define the regions inside and outside the $\mathrm{BH}$ because a distinction of the total system into two systems, say environment (I) and $B H$ (II), naturally emerges in the way just depicted. With this in mind, in what follows we shall nonetheless refer to system I as outside and to system II as inside. It is a worthy remark that other authors do use the geometric notions of exterior and interior of $\mathrm{BHs}$ even at fundamental levels [29]. Even though this can be justified, see, e.g., [4], and permits one to produce meaningful models, see, e.g., [39], our approach does not require it. The Hilbert space of 
physical states is then built as a subspace of a larger tensor product (kinematical) Hilbert space

$$
\mathcal{H} \subseteq \mathcal{H}_{\mathrm{I}} \otimes \mathcal{H}_{\mathrm{II}}
$$

We now assume that such a Hilbert space can be constructed with the methods of second quantization. This provides a language contiguous to the language of QFT, which should be recovered in some limit. Therefore, $\mathrm{BH}$ and environment modes will be described by two sets of creation and annihilation operators, which satisfy the usual canonical anticommutation relations

$$
\left\{\chi_{\tau n}, \chi_{\tau^{\prime} n^{\prime}}^{\dagger}\right\}=\delta_{\tau, \tau^{\prime}} \delta_{n n^{\prime}}
$$

with $n, n^{\prime}=1, \ldots, N, \tau=\mathrm{I}$, II, and all other anticommutators equal to zero. Then, we introduce the simplified notation

$$
\chi_{\mathrm{I} n}=a_{n} \otimes 1 \mathrm{I}_{\mathrm{II}} \equiv a_{n}, \quad \chi_{\mathrm{II} n}=1 \mathrm{I}_{\mathrm{I}} \otimes b_{n} \equiv b_{n} .
$$

We suppose that the initial state is

$$
|0, N\rangle \equiv|0,0, \ldots, 0\rangle_{\mathrm{I}} \otimes|1,1, \ldots, 1\rangle_{\mathrm{II}}
$$

where both kets, I and II, have $N$ entries and

$$
|1,1, \ldots, 1\rangle_{\mathrm{II}}=b_{1}^{\dagger} b_{2}^{\dagger} \ldots b_{N}^{\dagger}|0,0, \ldots, 0\rangle_{\mathrm{II}} .
$$

The state in Eq. (6) represents the $\mathrm{BH}$ at the beginning of the evaporation process with all the slots occupied by free $X$ ons. Although the $X$ ons, during the evaporation, are progressively arranged into less fundamental structures (and hence no longer are the d.o.f to be used for the emergent description), we keep our focus on them. For us this "transmutation" only helps in identifying what to call "inside" and what to call "outside" so that evaporation is the process that moves the Xons from II to I. In this way, the final state (for which there are no free Xons left, as they all recombined to form fields and spacetime), has the form

$$
|N, 0\rangle \equiv|1,1, \ldots, 1\rangle_{\mathrm{I}} \otimes|0,0, \ldots, 0\rangle_{\mathrm{II}}
$$

where

$$
|1,1, \ldots, 1\rangle_{\mathrm{I}}=a_{1}^{\dagger} a_{2}^{\dagger} \ldots a_{N}^{\dagger}|0,0, \ldots, 0\rangle_{\mathrm{I}} .
$$

In order to construct a state of the system compatible with the previous assumptions, let us consider the evolved operators as

$$
\begin{aligned}
& c_{n}(\sigma)=e^{i \psi_{n}}\left(b_{n} \cos \sigma+a_{n} e^{-i \varphi_{n}} \sin \sigma\right), \\
& d_{n}(\sigma)=e^{i \psi_{n}}\left(e^{-i \varphi_{n}} a_{n} \cos \sigma-b_{n} \sin \sigma\right),
\end{aligned}
$$

where on $\sigma$ we shall comment soon. Equations (10) and (11) define a canonical transformation

$$
\left\{c_{n}(\sigma), c_{m}^{\dagger}(\sigma)\right\}=\left\{d_{n}(\sigma), d_{m}^{\dagger}(\sigma)\right\}=\delta_{n m} .
$$

We thus get the evolution of the initial state (6) as

$$
\begin{aligned}
|\Psi(\sigma)\rangle & \equiv \prod_{n=1}^{N} c_{n}^{\dagger}(\sigma)|0\rangle_{\mathrm{I}} \otimes|0\rangle_{\mathrm{II}} \\
& =\prod_{n=1}^{N} e^{-i \psi_{n}}\left(b_{n}^{\dagger} \cos \sigma+a_{n}^{\dagger} e^{i \varphi_{n}} \sin \sigma\right)|0\rangle_{\mathrm{I}} \otimes|0\rangle_{\mathrm{II}}
\end{aligned}
$$

Strictly speaking, $\sigma$ should be regarded as a discrete parameter, counting the free Xons that leave the $\mathrm{BH}$, according to the picture described above (see also the discussion in the next section). Nonetheless, in order to simplify computations, and with no real loss of generality, we use the continuous approximation. Given our initial state [Eq. (6)] and final state [Eq. (8)], $\sigma$ can be seen as an interpolating parameter describing the evolution of the system from $\sigma=0$ corresponding to the beginning of the evaporation till $\sigma=\pi / 2$, corresponding to complete evaporation.

Let us also notice that the linear canonical transformation defined in Eqs. (10) and (11) is very general, given the requests. In fact, if we mix creation and annihilation operators, $c_{n}(\sigma) \sim\left(a_{n}+b_{n}^{\dagger}\right)$, one cannot interpolate Eqs. (6) and (8). Furthermore, the choice of phases introduced does not affect any of the results presented. This is a consequence of the fact that we are working with two types of modes (BH and environment). If we had more than two, we would have to deal with one or more physical phases, as is well known in quark and neutrino physics [45]. We can thus safely set $\varphi_{n}=0=\psi_{n}$.

With our choice of parameters, the state (13) can also be written as

$$
|\Psi(\sigma)\rangle=\prod_{i=1}^{N} \sum_{n_{i}=0,1} C_{i}(\sigma)\left(a_{i}^{\dagger}\right)^{n_{i}}\left(b_{i}^{\dagger}\right)^{1-n_{i}}|0\rangle_{\mathrm{I}} \otimes|0\rangle_{\mathrm{II}},
$$

with $C_{i}=(\sin \sigma)^{n_{i}}(\cos \sigma)^{1-n_{i}}$. This form would suggest the following generalization

$$
|\Phi(\sigma)\rangle=\prod_{i=1}^{N} \sum_{n_{i}, m_{i}=0,1} D_{i}(\sigma)\left(a_{i}^{\dagger}\right)^{n_{i}}\left(b_{i}^{\dagger}\right)^{m_{i}}|0\rangle_{\mathrm{I}} \otimes|0\rangle_{\mathrm{II}},
$$

with $D_{i}=(\sin \sigma)^{n_{i}}(\cos \sigma)^{m_{i}}$. However, we easily compute

$$
\begin{aligned}
|\Phi(0)\rangle= & \left|0_{1}, \ldots, 0_{N}\right\rangle_{\mathrm{II}} \otimes\left|1_{1}, \ldots, 1_{N}\right\rangle_{\mathrm{II}} \\
& +\left|0_{1}, \ldots, 0_{N}\right\rangle_{\mathrm{II}} \otimes\left|0_{1}, \ldots, 0_{N}\right\rangle_{\mathrm{II}},
\end{aligned}
$$


which is incompatible with our boundary condition (6). In order to enforce the latter, we need to impose the constraint $m_{i}=1-n_{i}$.

\section{ENTROPY OPERATORS, PAGE CURVE, AND THE BEKENSTEIN BOUND}

The Hilbert space of physical states has the dimension

$$
\Sigma \equiv \operatorname{dim} \mathcal{H}=2^{N} .
$$

The state defined in Eq. (13) is an entangled state. This is due to the fact that $c_{n}^{\dagger}(\sigma)$ cannot be factorized as $a_{n}$ and $b_{n}$ in Eq. (5), i.e., it cannot be written as $c_{n}^{\dagger}=A_{\mathrm{I}} \otimes B_{\mathrm{II}}$, where $A_{\mathrm{I}}\left(B_{\mathrm{II}}\right)$ acts only on $\mathcal{H}_{\mathrm{I}}\left(\mathcal{H}_{\mathrm{II}}\right)$.

To quantify such entanglement, we define the entropy operator for environment modes as in TFD [19,20,22]

$$
S_{\mathrm{I}}(\sigma)=-\sum_{n=1}^{N}\left(a_{n}^{\dagger} a_{n} \ln \sin ^{2} \sigma+a_{n} a_{n}^{\dagger} \ln \cos ^{2} \sigma\right) .
$$

We also define the entropy operator for $\mathrm{BH}$ modes in a rather unconventional way:

$S_{\text {II }}(\sigma)=-\sum_{n=1}^{N}\left(b_{n}^{\dagger} b_{n} \ln \cos ^{2} \sigma+b_{n} b_{n}^{\dagger} \ln \sin ^{2} \sigma\right)$.

The reason for such unconventional definition will be clear in the next section. For the moment, notice that we have two different operators for I and for II, but we see that since

$$
\left\langle a_{n}^{\dagger} a_{n}\right\rangle_{\sigma}=\sin ^{2} \sigma=1-\left\langle b_{n}^{\dagger} b_{n}\right\rangle_{\sigma},
$$

then

$$
\begin{aligned}
\mathcal{S}_{\mathrm{I}}(\sigma) & \equiv\left\langle S_{\mathrm{I}}(\sigma)\right\rangle_{\sigma} \\
& =-N\left(\sin ^{2} \sigma \ln \sin ^{2} \sigma+\cos ^{2} \sigma \ln \cos ^{2} \sigma\right) \\
& =\left\langle S_{\mathrm{II}}(\sigma)\right\rangle_{\sigma} \equiv \mathcal{S}_{\mathrm{II}}(\sigma),
\end{aligned}
$$

where $\langle\ldots\rangle_{\sigma} \equiv\langle\Psi(\sigma)|\ldots| \Psi(\sigma)\rangle$. Therefore, the averages of the operators coincide, as it must be for a bipartite system. This entropy is the entanglement entropy between environment and $\mathrm{BH}$ when the system evolves. Remarkably, it has a behavior in many respects similar to that of the Page curve [31], as shown in Fig. 1.

The maximum value is

$$
\mathcal{S}_{\max }=N \ln 2=\ln \Sigma,
$$

so that

$$
\Sigma=e^{\mathcal{S}_{\max }} .
$$

As we see here through Eq. (23) [that is the analogue of Eq. (1)], in our model $\operatorname{dim} \mathcal{H}$ is related to the maximal

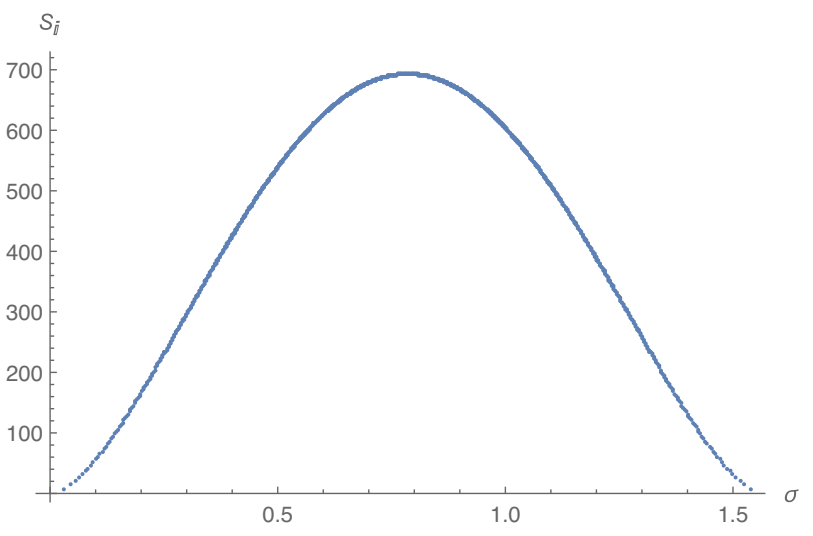

FIG. 1. The von Neumann entropy as a function of $\sigma$ in the case $N=1000$.

entanglement (von Neumann) entropy of the environment with the BH (and, of course, vice versa). This happens exactly when the modes have half probability to be inside and half probability to be outside the $\mathrm{BH},{ }^{1}$ and then a large amount of bits are necessary to describe the system. Thus, the system has an intrinsic way to know how big is the physical Hilbert space, hence to know how big is the BH at the beginning of the evaporation: when the maximal entanglement is reached, that value of the entropy $\mathcal{S}_{\max }$ tells how big was the original $\mathrm{BH}$. Hence, $\mathcal{S}_{\max }$ must be some function of $\mathcal{M}_{0}, \mathcal{Q}_{0}, \mathcal{J}_{0}$, i.e., the initial mass, charge, or angular momentum of the $\mathrm{BH}$.

This maximal entropy bound is obtained here as a mere consequence of the finiteness of the fermionic fundamental d.o.f, hence of a Pauli principle. No geometric notions (distance, area, Planck length, etc.) are employed. When such notions are eventually introduced, this bound must correspond to the Bekenstein bound. In other words, the necessary dynamical map connecting the Xons to fields and geometry will be introduced in such a way that this fundamental nongeometrical bound becomes the emergent geometrical Bekenstein bound. A brief discussion on the dynamical map is offered in the last section.

Therefore, for a full identification of $\mathcal{S}_{\max }$ with $\mathcal{S}_{\mathrm{BH}}$ we need more than what we have here. In particular, we need the concept of area that, somehow, is what has been evoked in LQG $[15,16]$ when in (22) one identifies

$$
N \equiv \frac{\mathcal{A}}{4 \pi \gamma l_{P}^{2} \sqrt{3}}
$$

where $\gamma$ is the Immirzi parameter. We shall comment more on this later.

We want now to bring into the picture the two missing pieces: how the entropy of the $\mathrm{BH}$, that should always decrease, and the entropy of the environment, that should

\footnotetext{
${ }^{1}$ Recall that we have an intrinsic nongeometric notion of the partition into inside/outside.
} 
always increase (hence, can be related to a standard thermodynamical entropy), actually evolve in our model. To this end, let us introduce the following number operators:

$$
\hat{N}_{\mathrm{I}}=\sum_{n=1}^{N} a_{n}^{\dagger} a_{n}, \quad \hat{N}_{\mathrm{II}}=\sum_{n=1}^{N} b_{n}^{\dagger} b_{n},
$$

that count the number of modes of the radiation and the number of modes of the $\mathrm{BH}$, respectively. Although it should be clear from the above example, it is nonetheless important to stress now again that, in our formalism, the full kinematical Hilbert spaces associated to both sides have fixed dimensions $\left(\operatorname{dim} \mathcal{H}_{\mathrm{I}}=\operatorname{dim} \mathcal{H}_{\mathrm{II}}=2^{N}\right)$, while only a subspace $\mathcal{H} \subseteq \mathcal{H}_{\mathrm{I}} \otimes \mathcal{H}_{\text {II }}$ such that $\operatorname{dim} \mathcal{H}=2^{N}$ is the one of physical states. Note that $\mathcal{H}$ cannot be factorized, and this is the origin of $\mathrm{BH} /$ environment entanglement.

Nonetheless, one could think that the physical Hilbert spaces of the two subsystems have to take into account only the number of modes truly occupied at any given stage of the evaporation. Hence, the actual dimensions would be $2^{N_{\mathrm{I}}(\sigma)}$ and $2^{N_{\mathrm{II}}(\sigma)}$, where one easily finds that

$$
N_{\mathrm{I}}(\sigma) \equiv\left\langle\hat{N}_{\mathrm{I}}\right\rangle_{\sigma}=N \sin ^{2} \sigma
$$

and

$$
\begin{aligned}
N_{\text {II }}(\sigma) & \equiv\left\langle\hat{N}_{\text {II }}\right\rangle_{\sigma} \\
& =N-N_{\mathrm{I}}(\sigma)=N \cos ^{2} \sigma .
\end{aligned}
$$

Recall that $\sigma$ is, in fact, a discrete parameter essentially counting the diminishing number of free Xons (as said earlier and shown in greater detail later).

In other words, when we take this view, the partition into I and II becomes in all respects similar to the one of Page [31], that is

$$
2^{N}=2^{N_{\mathrm{II}}(\sigma)} \times 2^{N_{\mathrm{I}}(\sigma)} \equiv n \times m,
$$

with $n=2^{N}, 2^{N-1}, \ldots, 1$ and $m=1, \ldots, 2^{N-1}, 2^{N}$, while $\sigma$ runs in discrete steps in the interval $[0, \pi / 2]$. Number fluctuations, which makes it necessary to invoke the entire Hilbert space $\mathcal{H}$ at each stage, represent a measure of entanglement of these modes, as we shall see below. It is then natural to define the Bekenstein entropy as

$$
\mathcal{S}_{\mathrm{BH}} \equiv \ln n=N \ln 2 \cos ^{2} \sigma,
$$

and the environment entropy ${ }^{2}$ as

$$
\mathcal{S}_{\text {env }} \equiv \ln m=N \ln 2 \sin ^{2} \sigma .
$$

\footnotetext{
${ }^{2}$ We could also call it thermodynamical entropy in comparison with the nomenclature of Ref. [31].
}

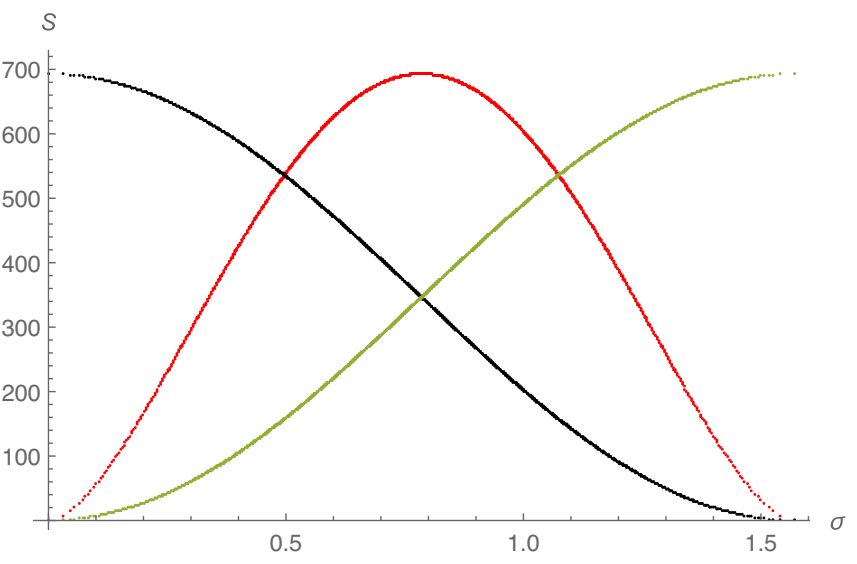

FIG. 2. Here we plot: $\mathcal{S}_{\mathrm{BH}}$ in black, monotonically decreasing; $\mathcal{S}_{\text {env }}$ in green, monotonically increasing; $\mathcal{S}_{\mathrm{I}}$ in red with a Pagelike behavior. Note that the maximal value of $\mathcal{S}_{\mathrm{I}}, S_{\max }$ coincides with the initial $\mathrm{BH}$ entropy, as well as with the final environment entropy, as inferred in the text. The plots are done for $N=1000$.

The plots of the three entropies $\mathcal{S}_{I}, \mathcal{S}_{\mathrm{BH}}$, and $\mathcal{S}_{\text {env }}$ are shown in Fig. 2 and must be compared with similar results of Ref. [36]. There are, though, two main differences worth stressing. First, we have a common single origin behind all involved entropies, as explained. Second, since the overall system here is based on the most fundamental entities, the curve for $\mathcal{S}_{I}$ cannot be always below the other two, as happens in [36], but its maximum $\mathcal{S}_{\max }$ must reach the starting point of $\mathcal{S}_{\mathrm{BH}}$ (and the ending point of $\mathcal{S}_{\text {env }}$ ). In our case, the inequality

$$
\mathcal{S}_{\mathrm{I}} \leq \mathcal{S}_{\mathrm{BH}}+\mathcal{S}_{\mathrm{env}}=\mathcal{S}_{\mathrm{max}},
$$

is always satisfied. Note also that the dynamics of our system is unitary because we keep our focus on the evaporated $X$ ons and not on the emerging structures, as was done in [1]. Hence, we are not in the position here to spot the relic entanglement between fields and spacetime that would make the curves for $\mathcal{S}_{\mathrm{BH}}$ and $\mathcal{S}_{I}$ end at a nonzero value. The latter is precisely the source of the information lost in the quasiparticle picture of [1]. Whether or not this formally unitary evolution is physically tenable at the emergent level, and the impact of this on the validity of the Stone-von Neumann uniqueness theorem [20,46,47] in a quantum system with a finite-dimensional Hilbert space, is under scrutiny in ongoing research [48]. Recently, the relation between unitarity and the existence of a maximal entropy has been also investigated in Ref. [49].

It is worth noticing that the total entropy $\mathcal{S}_{\mathrm{BH}}+\mathcal{S}_{\text {env }}$ in Eq. (31) is a constant along the evolution parameter $\sigma$ : it is tempting to recognize this as a generalized second law (GSL), saturated in this case, due to the fact that we are probing the fundamental non-coarse-grained level of the $X$ ons. However, we stress again here that $\sigma$ cannot be directly related to time evolution yet and hence a 
comparison with a GSL can be premature. Instead, one could expect that a proper GSL should arise from the coarse-grained description given by a dynamical map, something that was already hinted to in [1] due to the presence of the final residual entropy.

It is perhaps worthwhile to stress that Eq. (21) represents exactly a von Neumann entropy. We can write the density matrix $\rho(\sigma)=|\Psi(\sigma)\rangle\langle\Psi(\sigma)|$ and evaluate the reduced density matrices $\rho_{\mathrm{I}}=\operatorname{Tr}_{\mathrm{II}} \rho$ and $\rho_{\mathrm{II}}=\operatorname{Tr}_{\mathrm{I}} \rho$ [50]. We can then easily check that (see Appendix)

$$
\begin{aligned}
\mathcal{S}_{\mathrm{I}}(\sigma) & =-\operatorname{Tr}_{\mathrm{I}}\left(\rho_{\mathrm{I}}(\sigma) \ln \rho_{\mathrm{I}}(\sigma)\right) \\
& =-N\left(\cos ^{2} \sigma \ln \cos ^{2} \sigma+\sin ^{2} \sigma \ln \sin ^{2} \sigma\right) \\
& =-\operatorname{Tr}_{\mathrm{II}}\left(\rho_{\mathrm{II}}(\sigma) \ln \rho_{\mathrm{II}}(\sigma)\right)=\mathcal{S}_{\mathrm{II}}(\sigma) .
\end{aligned}
$$

Let us now consider some simple cases

(i) If $N=1$, then we have

$$
|\Psi(\sigma)\rangle=\cos \sigma|0\rangle_{\mathrm{I}} \otimes|1\rangle_{\mathrm{II}}+\sin \sigma|1\rangle_{\mathrm{I}} \otimes|0\rangle_{\mathrm{II}} .
$$

In general, this is an entangled state whose maximal entanglement is reached for $\sigma=\pi / 4$ :

$$
|\Psi(\pi / 4)\rangle=\frac{1}{\sqrt{2}}\left(|0\rangle_{\mathrm{I}} \otimes|1\rangle_{\mathrm{II}}+|1\rangle_{\mathrm{I}} \otimes|0\rangle_{\mathrm{II}}\right) .
$$

(ii) For $N=2$ we have

$$
\begin{aligned}
|\Psi(\sigma)\rangle= & \cos ^{2} \sigma\left|0_{1} 0_{2}\right\rangle_{\mathrm{I}} \otimes\left|1_{1} 1_{2}\right\rangle_{\mathrm{II}} \\
& +\sin ^{2} \sigma\left|1_{1} 1_{2}\right\rangle_{\mathrm{I}} \otimes\left|0_{1} 0_{2}\right\rangle_{\mathrm{II}} \\
& +\cos \sigma \sin \sigma\left|0_{1} 1_{2}\right\rangle_{\mathrm{I}} \otimes\left|1_{1} 0_{2}\right\rangle_{\mathrm{II}} \\
& +\cos \sigma \sin \sigma\left|1_{1} 0_{2}\right\rangle_{\mathrm{I}} \otimes\left|0_{1} 1_{2}\right\rangle_{\mathrm{II}}
\end{aligned}
$$

It is then clear that the mean number (20) represents the probability of the $n$th mode to "leave the $\mathrm{BH}$ phase" (to go from II to I).

As mentioned earlier, $\sigma$ for us is a continuous approximation of a discrete parameter that counts the Xons transmuting from being free (in the $\mathrm{BH}$, II) to being arranged into fields and spacetime (that is what happens, eventually, in I). Now we can formalize that statement by inverting Eq. (26) and getting

$$
\sigma\left(N_{\mathrm{I}}\right)=\arcsin \sqrt{\frac{N_{\mathrm{I}}}{N}}
$$

When $N_{I}$ is constrained to be an integer $N_{I}=m$, the $\sigma\left(N_{\mathrm{I}}\right)=\sigma_{m}$ is discretized. Therefore, the evolution parameter is just a way of counting how many modes jumped out. It cannot be regarded as time, which should emerge, like space, at low energy from $X$ ons dynamics. The von Neumann entropy as a function of $\sigma=\sigma_{m}$ is reported in Fig. 1.

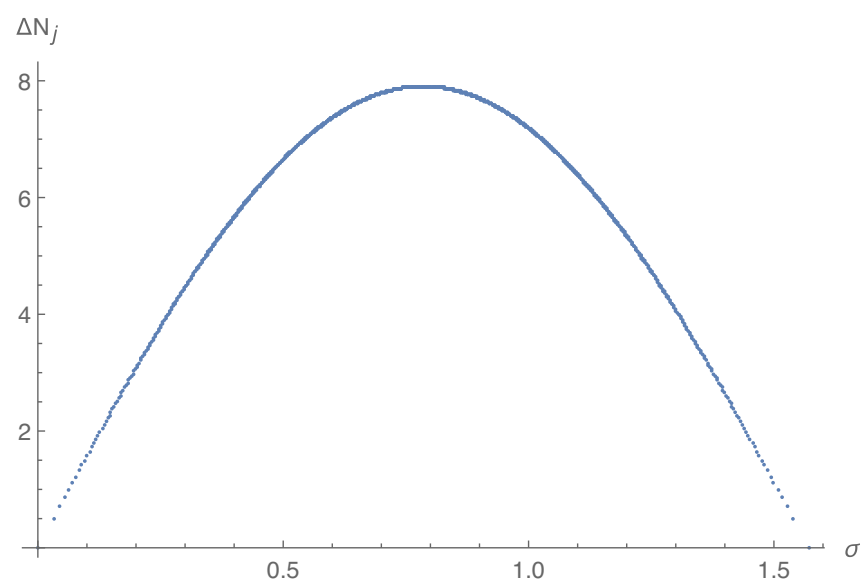

FIG. 3. $\Delta N_{j}$ as a function of $\sigma$ in the case $N=1000$.

We could then expect that at each step the number of $\mathrm{BH} /$ environment modes was fixed. What is the meaning of fluctuations of $\hat{N}_{\text {I }}$ and $\hat{N}_{\text {II }}$ on $|\psi(\sigma)\rangle$ ? A direct computation shows that

$$
\Delta N_{\mathrm{I}}(\sigma)=\Delta N_{\mathrm{II}}(\sigma)=\frac{\sqrt{N} \sin (2 \sigma)}{2},
$$

where $\Delta N_{j} \equiv \sqrt{\left\langle\hat{N}_{j}^{2}\right\rangle_{\sigma}-\left\langle\hat{N}_{j}\right\rangle_{\sigma}^{2}}$ is the standard deviation of $\hat{N}_{j}$ on $|\Psi(\sigma)\rangle$. In agreement with the general results of Ref. [51], Fig. 3 clearly shows that this is a measure of the entanglement. Moreover, for $N=1,\left(\Delta N_{j}(\sigma)\right)^{2}$ is proportional to the linear entropy or impurity

$$
\left(\Delta N_{j}(\sigma)\right)^{2}=2 S_{L}^{j}(\sigma), \quad j=\mathrm{I}, \mathrm{II},
$$

defined as $[50,52]$

$$
S_{L}^{j} \equiv 1-\operatorname{Tr} \rho_{j}^{2}
$$

Note that $\Delta N_{j}$ can be easily discretized as explained above. We finally turn our attention to the generator of the canonical transformations in (10) and (11) (with our choice of parameters)

$$
\begin{gathered}
a_{n}(\sigma) \equiv d_{n}(\sigma)=e^{-i \sigma G} a_{n}(0) e^{i \sigma G}, \\
b_{n}(\sigma) \equiv c_{n}(\sigma)=e^{-i \sigma G} b_{n}(0) e^{i \sigma G},
\end{gathered}
$$

where one can easily check that

$$
G=i \sum_{n=1}^{N}\left(a_{n}^{\dagger} b_{n}-b_{n}^{\dagger} a_{n}\right) .
$$

With the above recalled limitations, the existence of such unitary generators is guaranteed by the Stone-von Neumann theorem and, in the general meaning of [16], it can be seen to enter the Wheeler-DeWitt equation 


$$
H|\Psi(\sigma)\rangle=0,
$$

with $H \equiv i \partial_{\sigma}-G$. This constrains the kinematical Hilbert space $\mathcal{H}_{\mathrm{I}} \otimes \mathcal{H}_{\mathrm{II}}$ to the physical Hilbert space $\mathcal{H}$ as previously extensively commented. Let us remark that for $\sigma=\sigma_{m}$, Eq. (43) becomes a linear difference (recursion) equation.

\section{CONNECTION WITH DISSIPATIVE SYSTEMS}

In the previous section we have shown how our toy model possesses a Page-like behavior for entanglement entropy and this can be easily computed by means of the TFD entropy operator. We now ask if this is a mere coincidence or if the connection with TFD can be made more precise.

Let us perform the canonical transformation

$$
A_{n}=a_{n}, \quad B_{n}=b_{n}^{\dagger} .
$$

This is not a special Bogoliubov transformation [53]. In fact, this transformation can be obtained from

$$
\begin{aligned}
& A_{n}=a_{n} \cos \theta_{n}-b_{n}^{\dagger} \sin \theta_{n}, \\
& B_{n}=b_{n}^{\dagger} \cos \theta_{n}+a_{n} \sin \theta_{n},
\end{aligned}
$$

for $\theta_{n}=0$. Then it is not connected with the identity. However, we can still define vacua in the new representation

$$
A_{n}|0\rangle_{A}=B_{n}|0\rangle_{B}=0 .
$$

One can check that

$$
\begin{gathered}
|0\rangle_{A}=|0\rangle_{\mathrm{I}}, \\
|0\rangle_{B}=\left|1_{1} 1_{2} \ldots 1_{N}\right\rangle_{\mathrm{II}} .
\end{gathered}
$$

The second relation follows from the fact that $\left(b_{n}^{\dagger}\right)^{2}=0$. Therefore, the generator (42) becomes

$$
G=i \sum_{n=1}^{N}\left(A_{n}^{\dagger} B_{n}^{\dagger}-B_{n} A_{n}\right) .
$$

This is nothing but the (fermionic version of the) interaction Hamiltonian of quantum dissipative systems, as introduced in Ref. [54] (see also [55]). This operator noticeably coincides with the generator of a special Bogoliubov transformation. Therefore, $|\Psi(\sigma)\rangle$ has a TFD-vacuum-like structure $[19,20]$

$$
\begin{aligned}
|\Psi(\sigma)\rangle & =\prod_{n=1}^{N}\left(\cos \sigma+\sin \sigma A_{n}^{\dagger} B_{n}^{\dagger}\right)|0\rangle_{A} \otimes|0\rangle_{B} \\
& =e^{-\frac{1}{2} S_{A}(\sigma)}|I\rangle,
\end{aligned}
$$

with $|I\rangle=\exp \left(\sum_{n=1}^{N} A_{n}^{\dagger} B_{n}^{\dagger}\right)|0\rangle_{A} \otimes|0\rangle_{B}$, and the entropy operators

$$
\begin{aligned}
& S_{A}=-\sum_{n=1}^{N}\left(A_{n}^{\dagger} A_{n} \ln \sin ^{2} \sigma+A_{n} A_{n}^{\dagger} \ln \cos ^{2} \sigma\right), \\
& S_{B}=-\sum_{n=1}^{N}\left(B_{n}^{\dagger} B_{n} \ln \sin ^{2} \sigma+B_{n} B_{n}^{\dagger} \ln \cos ^{2} \sigma\right) .
\end{aligned}
$$

Therefore, through (44), we have now the usual entropy operators of TFD [19,20,22] to be compared with the unusual definitions of (19): $S_{\mathrm{I}}=S_{A}$ and $S_{\mathrm{II}}=S_{B}$.

The physical picture here is that, when the system evolves, a pair of $A$ and $B$ particles is created. The $\mathrm{B}$-modes enter into the $\mathrm{BH}$, annihilating $\mathrm{BH}$ modes, while the A-modes form the environment. This mechanism is heuristically the same as the one proposed by Hawking [18] and, lately, formalized via the tunneling effect [56].

The $A$ - and $B$-modes do not discern explicitly fields and geometric d.o.f. However, taking the view of Ref. [1], some d.o.f are indeed responsible for the reduction of the BH's horizon area during the evaporation and annihilators of geometric modes can be defined. In order to make this idea more precise, we can decompose $A_{n}$ in their geometric $(G)$ and field $(F)$ parts as follows:

$$
A_{n}=\sum_{k}\left(g_{k, n} A_{G, n}^{k} \otimes 1 \mathrm{I}_{F, n}+f_{k, n} 1 \mathrm{I}_{G, n} \otimes A_{F, n}^{k}\right),
$$

where $k$ labels the emergent modes. Equation (54) can be regarded as a dynamical (Haag) map at linear order [20]. The full dynamical map-available once the quantum theory of gravity is specified-should connect the fundamental d.o.f to the emergent notions of geometry and fields. The coefficients of the map should then lead to the Hawking thermal behavior of the latter at the emergent level. Note that the action of $A_{n}^{\dagger}$ on $|0\rangle_{A}$, creates both a matter mode and a geometric mode outside the horizon: the region of spacetime surrounding the $\mathrm{BH}$ and available to an external observer increases because the horizon area decreases.

Let us remark that, in this picture, a relationship of the form (54) makes sense only for $A$-modes (or equivalently $a$-modes) and not for $B$-modes (or equivalently $b$-modes). In fact, by definition, the former are the ones which rearrange to form matter and geometry, in contrast with the latter which describes free Xons. As previously stated, such a distinction is at the basis of our intrinsic notion of interior/exterior of the $\mathrm{BH}$. A more detailed analysis of this issue will be addressed in a forthcoming work.

It is only once we have the notion of area that we could try to fix $N$ in terms of the BH parameters $\mathcal{M}_{0}, \mathcal{Q}_{0}, \mathcal{J}_{0}$, and of the Planck length $l_{P}$. In fact, as remarked in Refs. [57,58], a quantum of BH horizon area measures 


$$
\Delta A=\alpha l_{P}^{2},
$$

where $\alpha$ is a constant to be fixed. Therefore, the BH entropy assumes the form ${ }^{3}$

$$
\mathcal{S}_{\mathrm{BH}}=\frac{\alpha N}{4},
$$

where $N$ is the number of Planck cells that, as remarked in the Introduction, would correspond to the number of our quantum levels/slots.

The value of $\alpha$ was fixed to $\alpha=4 \ln 3$ in Ref. [57], by means of arguments based on Bohr's correspondence principle, and to $\alpha=8 \pi$ in Ref. [58], by means of arguments based on the BH quasinormal modes. In our case, a comparison between (56) and (22) gives

$$
\alpha=4 \ln 2 .
$$

This value agrees with the condition $\alpha=4 \ln k$ with $k$ integer, which was proposed in Ref. [57], in order to constrain the number of microstates $\Sigma$ to an integer [see Eq. (23)]. Therefore, our model gives $k=2$. A comparison with the standard Bekenstein formula, when $\mathcal{Q}_{0}=0=\mathcal{J}_{0}$, gives

$$
N=\frac{4 \pi \mathcal{M}_{0}^{2}}{l_{P}^{2} \ln 2},
$$

which, of course, is again Eq. (24) when the area is expressed in terms of $\mathcal{M}_{0}$ and the Immirzi parameter is fixed to $\ln 2 /(\sqrt{3} \pi)$. In fact, both derivations-ours and LQG'srely on the identification of the entropy with $S_{\mathrm{BH}}$. Clearly, a more detailed analysis, beyond these qualitative arguments, will be possible only once a complete dynamical map will be available.

\section{CONCLUSIONS}

We assumed here that the d.o.f of a $\mathrm{BH}$ are finite in number and fermionic in nature and, hence obey a Pauli principle. Then, within the approach of second quantization, we naturally obtained that the $\mathrm{BH}$ evaporation is a dynamical mechanism producing a maximal entanglement entropy equal to the initial entropy of the BH. This phenomenon is an instance of the Bekenstein bound, obtained here with arguments that do not assume preexisting spatiotemporal concepts. Of course, for a full identification with the standard formulae (see, e.g., $[7,8]$ ), one needs to link geometrical concepts, such as elementary Planck cells, to such fundamental d.o.f.

We then showed that entanglement, Bekenstein, and environment (thermodynamic) entropies here are all naturally obtained in the same approach, based on an entropy operator whose structure is the one typical of TFD.

\footnotetext{
${ }^{3}$ This is equal to $\mathcal{S}_{\mathrm{BH}}$, defined in Eq. (29), only before the evaporation when $\mathcal{S}_{\mathrm{BH}}=\mathcal{S}_{\max }$.
}

Through such an operator, we have evaluated the von Neumann BH-environment entropy and noticeably obtained a Page-like evolution.

We finally have shown that the latter is a consequence of a duality between our model and a dissipativelike fermionic quantum system, and hence it has a natural TFD-like description.

Many directions for further research need be thoroughly explored, the most important being a reliable dynamical map from the fundamental modes to the emergent fields/ spacetime structures. This would pave the road for the investigation of important aspects of the emergent picture, such as the Hawking thermality of the field sector. Moreover, a geometric interpretation of the results presented here would allow one to investigate the discreteness of the spectrum of quasinormal modes, which is known to be related to the quantization of the BH horizon's area (see Ref. [58]). Anyway, even in the absence of a full dynamical map, we believe that our simple, although nontrivial, considerations are necessary to fully take into account the fascinating and far-reaching consequences of the Bekenstein bound.

\section{ACKNOWLEDGMENTS}

The authors would like to thank G. Vitiello, P. Lukeš, D. Lanteri, and L. Buoninfante for enlightening discussions. The authors profoundly thank Martin Scholtz, who sadly passed away, for his continuous inspiration. A. I. and L. S. are partially supported by UNiversity CEntre (UNCE) of Charles University (Prague, Czech Republic) Grant No. UNCE/SCI/013.

\section{APPENDIX: EQUIVALENCE OF TFD ENTROPY AND VON NEUMANN ENTROPY}

In this Appendix we explicitly show the equivalence of the TFD entropy of Eq. (21) with the von Neumann entropy of Eq. (32). We first present the computation in the simplest cases of Eqs. (33) and (35), and then we shall focus on the computation of the von Neumann entropy. Let us note that the expectation value of the TFD entropy operators [see Eq. (21)] immediately follows from Eq. (20).

(i) In the case $N=1$, the density matrix reads

$$
\begin{array}{r}
\rho(\sigma)=\cos ^{2} \sigma|0\rangle_{\mathrm{I}}|1\rangle_{\mathrm{IIII}}\left\langle\left. 1\right|_{\mathrm{I}}\left\langle 0\left|+\sin ^{2} \sigma\right| 1\right\rangle_{\mathrm{I}} \mid 0\right\rangle_{\mathrm{IIII}}\left\langle\left. 0\right|_{\mathrm{I}}\langle 1|\right. \\
+\frac{\sin (2 \sigma)}{2}\left(|0\rangle_{\mathrm{I}}|1\rangle_{\mathrm{IIII}}\left\langle\left. 0\right|_{\mathrm{I}}\langle 1|+| 1\rangle_{\mathrm{I}} \mid 0\right\rangle_{\mathrm{IIII}}\left\langle\left. 1\right|_{\mathrm{I}}\langle 0|\right),\right.
\end{array}
$$

where we omitted tensor product symbols. The reduced density matrices have the following form:

$$
\begin{gathered}
\rho_{\mathrm{I}}(\sigma)=\cos ^{2} \sigma|0\rangle_{\mathrm{II}}\left\langle 0\left|+\sin ^{2} \sigma\right| 1\right\rangle_{\mathrm{II}}\langle 1|, \\
\rho_{\mathrm{II}}(\sigma)=\cos ^{2} \sigma|1\rangle_{\mathrm{IIII}}\left\langle 1\left|+\sin ^{2} \sigma\right| 0\right\rangle_{\mathrm{IIII}}\langle 0| .
\end{gathered}
$$


Eq. (32) follows immediately from these expressions.

(ii) In the case $N=2$, we directly report the reduced density matrices:

$$
\begin{aligned}
\rho_{\mathrm{I}}(\sigma)= & \cos ^{4} \sigma|00\rangle_{\mathrm{II}}\left\langle 00\left|+\sin ^{4} \sigma\right| 11\right\rangle_{\mathrm{II}}\langle 11| \\
& +\frac{\sin ^{2} 2 \sigma}{4}\left(|01\rangle_{\mathrm{II}}\langle 01|+| 10\rangle_{\mathrm{II}}\langle 10|\right), \\
\rho_{\mathrm{II}}(\sigma)= & \cos ^{4} \sigma|11\rangle_{\mathrm{IIII}}\left\langle 11\left|+\sin ^{4} \sigma\right| 00\right\rangle_{\mathrm{IIII}}\langle 00| \\
& +\frac{\sin ^{2}(2 \sigma)}{4}\left(|01\rangle_{\mathrm{IIII}}\langle 01|+| 10\rangle_{\mathrm{IIII}}\langle 10|\right) .
\end{aligned}
$$

It follows that

$$
\begin{aligned}
\mathcal{S}_{\mathrm{I}}(\sigma)= & -\sin ^{4} \sigma \ln \sin ^{4} \sigma-\cos ^{4} \sigma \ln \cos ^{4} \sigma \\
& -2 \sin ^{2} \sigma \cos ^{2} \sigma \ln \left(\sin ^{2} \sigma \cos ^{2} \sigma\right) \\
= & \mathcal{S}_{\mathrm{II}}(\sigma) .
\end{aligned}
$$

By using the relations $\ln (a b)=\ln a+\ln b$ and $\cos ^{2} \sigma+\sin ^{2} \sigma=1$, we get

$$
\begin{aligned}
\mathcal{S}_{\mathrm{I}}(\sigma) & =-2\left(\sin ^{2} \sigma \ln \sin ^{2} \sigma+\cos ^{2} \sigma \ln \cos ^{2} \sigma\right) \\
& =\mathcal{S}_{\mathrm{II}}(\sigma),
\end{aligned}
$$

which is equal to Eq. (32) for $N=2$.

One could repeat similar computations for all $N$. However, it is simpler to use the correspondence of our model with a TFD/dissipative system via Eq. (44). As already known in TFD, the "thermal vacuum" can be rewritten in the form

$$
|\Psi(\sigma)\rangle=\sum_{n=0,1} \sqrt{w_{n}(\sigma)}|n\rangle_{A}|n\rangle_{B}
$$

where $\left|n_{A}\right\rangle_{A},\left|n_{B}\right\rangle$ are eigenstates of the number operators

$$
\hat{N}_{A}=\sum_{n=1}^{N} A_{n}^{\dagger} A_{n}, \quad \hat{N}_{B}=\sum_{n=1}^{N} B_{n}^{\dagger} B_{n} .
$$

Moreover, the coefficients $w_{n}$ are given by

$$
w_{n}(\sigma)=\prod_{j=1}^{N} C_{j}^{2}(\sigma)
$$

and $C_{j}$ were first introduced in Eq. (14). The density matrix thus reads

$$
\rho(\sigma)=\sum_{n=0,1} w_{n}(\sigma)|n\rangle_{A}|n\rangle_{B B}\left\langle\left. n\right|_{A}\langle n|,\right.
$$

from which the reduced density matrices are easily derived:

$$
\begin{aligned}
& \rho_{A}(\sigma)=\sum_{n=0,1} w_{n}(\sigma)|n\rangle_{A A}\langle n| \\
& \rho_{B}(\sigma)=\sum_{n=0,1} w_{n}(\sigma)|n\rangle_{B B}\langle n| .
\end{aligned}
$$

The von Neumann entropy reads [19,22,27]

$$
\mathcal{S}_{A}(\sigma)=\mathcal{S}_{B}(\sigma)=-\sum_{n=0,1} w_{n}(\sigma) \ln w_{n}(\sigma)
$$

Finally, Eq. (32) follows from substituting the explicit form of $w_{n}$ [cf. Eq. (A10)] into the last expression.
[1] G. Acquaviva, A. Iorio, and M. Scholtz, Ann. Phys. (Amsterdam) 387, 317 (2017).

[2] J. D. Bekenstein, Phys. Rev. D 23, 287 (1981); Found. Phys. 35, 1805 (2005).

[3] R. Feynman, R. Leighton, M. Sands, and M Gottlieb, The Feynman Lectures on Physics (Pearson/Addison-Wesley, Reading, MA, 2006); A. Iorio, J. Phys. Conf. Ser. 1275, 012013 (2019).

[4] N. Bao, S. M. Carroll, and A. Singh, Int. J. Mod. Phys. D 26, 1743013 (2017).

[5] J. D. Bekenstein, Sci. Am. 289, 58 (2003).

[6] D. Harlow, Rev. Mod. Phys. 88, 015002 (2016).

[7] H. Casini, Classical Quantum Gravity 25, 205021 (2008).
[8] R. Bousso, Rev. Mod. Phys. 74, 825 (2002).

[9] J. D. Bekenstein, Lett. Nuovo Cimento 4, 737 (1972).

[10] J. D. Bekenstein, Phys. Rev. D 7, 2333 (1973).

[11] S. W. Hawking, Phys. Rev. D 13, 191 (1976).

[12] W. H. Zurek, Phys. Rev. Lett. 49, 1683 (1982).

[13] D. N. Page, Phys. Rev. Lett. 50, 1013 (1983).

[14] W. Israel, Phys. Rev. 164, 1776 (1967); Commun. Math. Phys. 8, 245 (1968); B. Carter, Phys. Rev. Lett. 26, 331 (1971).

[15] A. Ashtekar, J. Baez, A. Corichi, and K. Krasnov, Phys. Rev. Lett. 80, 904 (1998).

[16] C. Rovelli, Quantum Gravity (Cambridge University Press, Cambridge, England, 2004); C. Rovelli and F. Vidotto, 
Covariant Loop Quantum Gravity: An Elementary Introduction to Quantum Gravity and Spinfoam Theory (Cambridge University Press, Cambridge, England, 2014).

[17] P. F. González-Díaz, Phys. Rev. D 27, 3042 (1983).

[18] S. W. Hawking, Nature (London) 248, 30 (1974); Commun. Math. Phys. 43, 199 (1975).

[19] Y. Takahashi and H. Umezawa, Collective Phenomena 2, 55 (1975); reprinted in Int. J. Mod. Phys. B 10, 1755 (1996).

[20] H. Umezawa, H. Matsumoto, and M. Tachiki, Thermo Field Dynamics And Condensed States (North-Holland, Amsterdam, 1982); H. Umezawa, Advanced Field Theory: Micro, Macro, and Thermal Physics (AIP, New York, 1993).

[21] W. Israel, Phys. Lett. 57A, 107 (1976).

[22] A. Iorio, G. Lambiase, and G. Vitiello, Ann. Phys. (Amsterdam) 309, 151 (2004).

[23] M. Martellini, P. Sodano, and G. Vitiello, Nuovo Cimento 48, 341 (1978).

[24] C. E. Laciana, Gen. Relativ. Gravit. 26, 363 (1994).

[25] J. M. Maldacena, J. High Energy Phys. 04 (2003) 021.

[26] M. Botta Cantcheff, A. L. Gadelha, D. F. Z. Marchioro, and D. L. Nedel, Eur. Phys. J. C 78, 105 (2018).

[27] M. Dias, D. L. Nedel, and C. R. Senise, arXiv:1910.11427.

[28] S. W. Hawking, Phys. Rev. D 14, 2460 (1976).

[29] D. N. Page, Phys. Rev. Lett. 44, 301 (1980).

[30] S. B. Giddings and W. M. Nelson, Phys. Rev. D 46, 2486 (1992).

[31] D. N. Page, Phys. Rev. Lett. 71, 3743 (1993).

[32] G. 't Hooft, Nucl. Phys. B, Proc. Suppl. 43, 1 (1995).

[33] S. W. Hawking, Phys. Rev. D 72, 084013 (2005).

[34] P. Hayden and J. Preskill, J. High Energy Phys. 09 (2007) 120.

[35] S. D. Mathur, Classical Quantum Gravity 26, 224001 (2009).

[36] D. N. Page, J. Cosmol. Astropart. Phys. 09 (2013) 028.

[37] J. Polchinski, The black hole information problem, New Frontiers in Fields and Strings: TASI 2015 Proceedings of the 2015 Theoretical Advanced Study Institute in Elementary Particle Physics (World Scientific, 2017), pp. 353-397, https://doi.org/10.1142/9789813149441_0006.

[38] M. Al Alvi, M. Majumdar, M. A. Matin, M. H. Rahat, and A. Roy, Phys. Lett. B 797, 134881 (2019).
[39] L. Piroli, C. Sünderhauf, and X. L. Qi, J. High Energy Phys. 04 (2020) 063.

[40] D. N. Page, Phys. Rev. Lett. 71, 1291 (1993).

[41] A. Almheiri, R. Mahajan, J. Maldacena, and Y. Zhao, J. High Energy Phys. 03 (2020) 149.

[42] S. Ryu and T. Takayanagi, Phys. Rev. Lett. 96, 181602 (2006).

[43] S. Sachdev, Phys. Rev. X 5, 041025 (2015).

[44] J. Maldacena and D. Stanford, Phys. Rev. D 94, 106002 (2016).

[45] M. Kobayashi and T. Maskawa, Prog. Theor. Phys. 49, 652 (1973); T. P. Cheng and L. Li, Gauge Theory of Elementary Particle Physics (Clarendon Press, Oxford, 1982).

[46] K. O. Friedrichs, Mathematical Aspects o! Quantum Theory of Fields (Interscience Publishers Inc., New York, 1953); H. Umezawa and G. Vitiello, Quantum Mechanics (Bibliopolis, Napoli, 1985); M. Blasone, P. Jizba, and L. Smaldone, Ann. Phys. (Amsterdam) 383, 207 (2017).

[47] A. Iorio and G. Vitiello, Mod. Phys. Lett. B 08, 269 (1994).

[48] G. Acquaviva, A. Iorio, and L. Smaldone (to be published).

[49] G. Dvali, arXiv:2003.05546.

[50] M. A. Nielsen and I. L. Chuang, Quantum Computation and Quantum Information (Cambridge University Press, Cambridge, England, 2000).

[51] A. A. Klyachko, B. Öztop, and A. S. Shumovsky, Phys. Rev. A 75, 032315 (2007).

[52] M. Blasone, P. Jizba, and G. Vitiello, Quantum Field Theory and Its Macroscopic Manifestations (World Scientific, London, 2011).

[53] J. P. Blaizot and G. Ripka, Quantum Theory of Finite Systems (MIT Press, Cambridge, 1986).

[54] E. Celeghini, M. Rasetti, and G. Vitiello, Ann. Phys. (N.Y.) 215, 156 (1992).

[55] A. Iorio and G. Vitiello, Ann. Phys. (N.Y.) 241, 496 (1995).

[56] M. K. Parikh and F. Wilczek, Phys. Rev. Lett. 85, 5042 (2000); L. Vanzo, G. Acquaviva, and R. Di Criscienzo, Classical Quantum Gravity 28, 183001 (2011).

[57] S. Hod, Phys. Rev. Lett. 81, 4293 (1998).

[58] M. Maggiore, Phys. Rev. Lett. 100, 141301 (2008). 